\title{
Current Insights: Evolving Principles and Controversies of Cancer Risk Assessment and Management of Hereditary Cancers
}

\author{
Presented by Robert Pilarski, MS, MSW, LGC; Jennifer M. Weiss, MD, MS; \\ Susan M. Domchek, MD; and Moderated by Tuya Pal, MD
}

\begin{abstract}
With the introduction of panel and direct-to-consumer testing, genetic testing has become commonplace in recent years, paving the way for both increased awareness around prevalent genetic cancer risks, and also an onslaught of misinformation. At the NCCN 2020 Virtual Annual Conference, Dr. Tuya Pal led a panel of experts in discussing the utility and difficulties associated with multigene testing, the emerging role of moderate-penetrance genes in defining risks for hereditary cancer, and the controversies associated with direct-to-consumer genetic testing services.

J Natl Compr Canc Netw 2020;18(7.5):964-967 doi: $10.6004 /$ jnccn.2020.5013
\end{abstract}

With the introduction of panel and direct-to-consumer (DTC) testing, genetic testing has become commonplace in recent years. Although this has paved the way for increased awareness around prevalent genetic cancer risks, it also allows for an onslaught of misinformation. At the NCCN 2020 Virtual Annual Conference, Tuya Pal, MD, Professor of Medicine, Vanderbilt-Ingram Cancer Center, led a panel of experts in discussing the utility and difficulties associated with multigene testing, the emerging role of moderate-penetrance genes in defining risks for hereditary cancer, and the controversies associated with direct-to-consumer genetic testing services.

\section{Promises and Problems of \\ Multigene Panel Testing}

Genetic testing options have changed significantly over the past several years. "Historically, we paid gene-by-gene for genetic testing, and because of that, we tested geneby-gene," said Robert Pilarski, MS, MSW, LGC, Associate Professor of Clinical Internal Medicine, The Ohio State University Comprehensive Cancer Center - James Cancer Hospital and Solove Research Institute. He noted some exceptions: BRCA1 and BRCA2 testing were offered together, as was testing for Lynch Syndrome genes. However, for the most part, patients were only tested for the gene most likely to explain the clinical presentation of their disease, with a price tag of approximately $\$ 1,000$ to $\$ 1,500$ per gene.
In recent years, however, single-gene testing has been rapidly supplanted by "panel testing," because it allows for analysis of multiple candidate genes at the same time, with a negligible increase in cost. "We've also realized as we've broadened testing that, in most cases, more than one gene could explain the cancer presentation in a patient or their family," he added.

However, multigene testing is not without its problems, including insurance coverage. For most insurers, coverage for testing is based on the patient meeting $B R C A$ or Lynch syndrome clinical testing criteria (which are often based on NCCN testing criteria). There are no criteria set by most insurers for genes other than these.

Insurers may also resist, and sometimes outright refuse to pay for panel testing, in part due to fears that it may lead to questionable management practices. If a mutation is found in a gene without proven management guidelines, patients or providers may still be inclined to conduct additional screening that is not evidence-based. As a result, testing laboratories may bill for any panel that includes $B R C A$ (or Lynch syndrome genes) as simply a BRCA (or Lynch) test, rather than billing for the broader panel, Mr. Pilarski added.

Another concern with multigene testing is clinical actionability. Not all genes being tested have clearly defined cancer risks; therefore, not all genes have established evidence-based management recommendations, or "clinical actionability," he explained. Further, not all 
genetic variants identified are clearly understood, and different types of mutations in certain genes may have differing clinical impact. Testing for multiple genes at one time also increases the likelihood of finding one or more variants of uncertain (or unknown) significance (which can be upsetting to some patients). Further complicating matters is the sheer number of various panel types and sizes that are now available from different labs.

\section{Pros and Cons of Panel Types}

The panelists noted 3 main categories of panel type: disease/organ-specific panels, guidelines-based panels, and comprehensive cancer panels. Disease/organ-specific panels only consider the genes that are known or suspected to cause the cancer(s) seen in the patient or family. These panels may miss syndromes that could later be correlated with the cancer of concern (eg, evidence is growing that Lynch syndrome may increase the risk for prostate cancer).

Guidelines-based panels only consider genes that have been determined to be clinically actionable. However, as Mr. Pilarski mentioned, this term is not clearly defined for all cancers, and this panel will likely leave out some candidate genes (eg, HOXB13 is an accepted prostate cancer gene without clear management recommendations, and therefore would be left off a prostate cancer guidelines-based panel).

The broadest category is comprehensive cancer panels. These panels identify the largest number of patients at risk for cancer and can find mutations in patients who didn't meet testing criteria for any given gene. "There is fairly clear evidence now that despite all of the effort that NCCN and other groups put into developing testing criteria, they still miss a significant number of patients who have mutations in genes," he said.

A downside of a comprehensive cancer panels is that the panel can identify mutations in genes unrelated to the cancer of concern. "If a family with hereditary risk for breast cancer is looking for answers, finding a mutation in a prostate or colon cancer gene may well be beneficial, but it's not giving them the answers to what they were concerned about," Mr. Pilarski said. This type of testing will also identify mutations in genes that are not clinically actionable (which may increase anxiety in the patient/family), and also has the highest likelihood of finding variants of uncertain significance.

Choosing a laboratory can be fairly complicated, he added, but considering some key factors can help providers narrow down the options and choose the best fit to address their own concerns and those of their patients (Figure 1).

\section{Role of Moderate-Penetrance Genes in Defining Hereditary Cancer Risks}

According to Jennifer M. Weiss, MD, MS, Associate Professor, and Director, UW Gastrointestinal Genetics Clinic, University of Wisconsin Carbone Cancer Center,
1. Are the laboratory directors experienced and appropriately trained?

2. Is the laboratory accredited?

3. What tests does the laboratory perform (limited or broad spectrum)

4. What genes are included on specific panels?

5. Testing methodologies/depth of coverage (aver vs $\mathrm{min}$ )/Sanger confirmation?

6. Variant classification program for variants of uncertain significance; reclassification policy

7. Test charges/costs to patients

8. Insurance/billing/financial assistance policies

9. What happens to a patient's genetic data and notification of reclassified variants of uncertain significance if a laboratory goes out of business?

Figure 1. Questions to use when considering a laboratory.

low- to moderate-risk genes tend to cause particular consternation within multigene panel testing. Moderatepenetrance genes are associated with a low to moderate risk of cancer (odds ratios, $\geq 2.0$ and $<5.0$ ), such as CHEK2 for breast cancer and colon cancer risk. To put this into context, commonly known high-risk genes for breast cancer are $B R C A 1$ and $B R C A 2$, with odds ratios $\geq 5.0$ ).

Another way to view moderate risk is through a framework by Slavin et al. ${ }^{1}$ "This framework talks about the combination of [moderate] penetrance, [moderate] actionability, and implications for family members," she said. "With these genes, organ-specific cancer risks are fairly well defined for at least one cancer site, but another cancer site might not be as well defined." For example, ATM causes an increased risk for breast cancer, but its association with pancreatic cancer risk is unclear.

This framework defines moderate actionability as having enough evidence for a gene-specific risk to supersede the empiric risk for enhanced surveillance for at least one cancer site, but possibly not for other sites. For example, increased breast cancer surveillance for PALB2 carriers is justified even in the absence of a family history of breast cancer. "But as far as implications for other family members, this is where it gets a little tricky," she said. "With moderate-risk genes, these implications may not be as straightforward." However, she added that it is important to keep in mind that, in reality, empiric risk modeling based on a patient's personal and family history of cancer may actually supersede the current knowledge of a gene-specific risk for cancer.

\section{Pros and Cons of Moderate-Penetrance Gene Inclusion}

The argument for including moderate-penetrance genes in multigene panels is that they may identify cancer risks not apparent based on an individual's family history alone; their inclusion will also likely facilitate the collection of data to better delineate cancer risks for these 
genes, and could help to refine guidelines for medical and surgical management.

Including moderate-penetrance genes in multigene panels also poses unique challenges, including difficulty around developing personalized management guidelines when the phenotypic spectrum and penetrance of these genes are less defined. "It is helpful if we have genespecific guidelines that will impact medical or surgical management for pathogenic and likely pathogenic variants in these genes," she noted. "However, it is not helpful if we do not have consensus recommendations for management."

Dr. Weiss also stressed that genetic testing recommendations in the NCCN Clinical Practice Guidelines in Oncology (NCCN Guidelines) change frequently. For example, heterozygous MUTYH pathogenic mutations are present in approximately $2 \%$ of the general population, and risk estimates for colorectal cancer are conflicting (no increased risk to $>$ twofold risk) ${ }^{1,2}$ The NCCN Guidelines for Genetic/Familial High-Risk Assessment: Colorectal for managing individuals with this gene mutation currently depend on whether or not the patient has a first- or second-degree relative with colorectal cancer. " ${ }^{3}$ These recommendations change as we get more data and evidence from individuals being diagnosed with these mutations in moderate-risk genes," she said.

\section{Recommendations for Clinical Practice}

For individuals with pathogenic mutations in moderate-risk genes, Dr. Weiss encourages thorough pretest counseling, especially around the possibility of unanticipated results, "as well as explaining what we do and don't know about how to manage these individuals if we find pathogenic mutations in these genes," she added. Also, consider the implications for cascade testing for moderate-penetrance genes. ${ }^{4}$ That is, is there evidence for enhanced surveillance for other family members or could this information impact family planning?

"Since the NCCN Guidelines are updated on a yearly basis, it is reasonable to have patients with pathogenic and likely pathogenic variants in moderate-risk genes follow up every 1 to 3 years, to see if the patient and family recommendations can be further clarified as more knowledge is obtained about the cancer risk," she said. Dr. Weiss also encourages risk-adapted screening for the management of individuals with variants in these genes.

"We know that risk based on family history, known exposures, lifestyle, and issues such as smoking and obesity can all help determine a personalized plan for individuals who have mutations in these moderate-risk genes," she said.

\section{Promises and Perils of Direct-To-Consumer Genetic Testing}

When it comes to direct-to-consumer (DTC) testing, according to Susan M. Domchek, MD, Professor, and Executive Director, Basser Center for BRCA, Abramson Cancer Center at the University of Pennsylvania, "the horse has long since left the barn."

Testing companies, to their credit, have increasingly provided content on their websites regarding education," she said "But it's worth stating that there's no requirement that anyone reads any of those educational materials." Patients can click "add to cart" without clicking anything else, and informed consent is technically "signed," but can be scrolled through quickly.

For all DTC approaches (Figure 2), the potential benefit is the expansion of access to genetic testing. "The idea here is that a healthcare provider is a barrier to access, not a facilitator, and that artificial things like guidelines get in the way of patients wanting to understand their genetic risk," she said. She also noted that in the patient-initiated, physician-mediated model, the physician is not usually the patient's own, but is generally provided by the company. Notably, there are data that the recommendation for genetic testing by one's own provider increases the likelihood of genetic testing; that is healthcare providers may be facilitators not barriers.

DTC testing is often associated with a perception of decreased cost. Cost may be lower for individuals without a family history of cancer who do not meet criteria for genetic testing and therefore would have to pay out of pocket, but DTC testing is not necessarily less expensive for those who do meet testing criteria; they often pay more for DTC testing than they would through traditional channels, she noted.

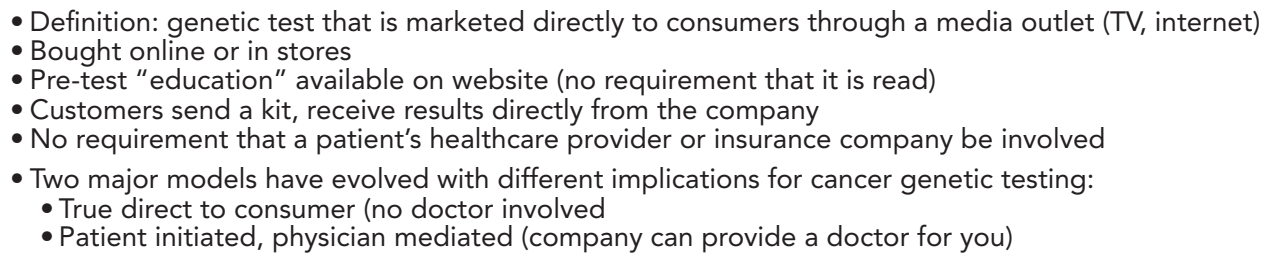

Figure 2. Direct-to-consumer genetic testing. 
Some have also suggested that DTC testing might improve testing disparities, because it is well known that underrepresented minorities are unlikely to undergo genetic testing even when personal and family history dictate that they should. However, according to Dr. Domchek, there is no evidence that this type of testing might balance those disparities. In fact, she said, it's possible that DTC testing might worsen them due to out of pocket cost considerations.

\section{DTC Models, Benefits, and Concerns}

According to Dr. Domchek, DTC testing such as 23andMe is limited in its ability to test for cancer susceptibility, and uses genotyping technology of selected variants, rather than full DNA sequencing. "This matters," she said, "because you will not get full sequencing of BRCA1 or BRCA2 or any other genes on the panel."

She also noted that even if a patient receives a positive result for a gene mutation, language on the company website states that results should be confirmed in a clinical setting before any medical action is taken. Dr. Domchek suggests that this only adds to the ambiguity around these results. "It's not clear whether people are coming to 23andMe for cancer testing or for ancestry and recreational testing, and they end up receiving some cancer testing along the way," she said. "I don't think we have good data on that yet."

Consumer-initiated models, conversely, do use nextgeneration sequencing, and these panels can include a large number of genes. However, the consumer typically has no control over the product. "Unlike the setting in which we are directly evaluating someone's family history and also eliciting their preferences for information, the consumer is ordering off of a menu and they have no control over what's on it," she said. This begs the question of whether or not most people really know what they're signing up for, and whether a physician-mediated panel is the right panel for a person's particular family history. ${ }^{5}$
According to Dr. Domchek, it remains abundantly unclear whether people understand what they're getting with DTC testing, be it limited information through DTC companies (ie, for $B R C A 1 / 2$, 23andMe tests only for 3 common Ashkenazi Jewish founder mutations), or potentially more than they bargained for with large consumer-initiated models that often conduct combined panels (encompassing much more than just cancer) ${ }^{6}$

Yet another issue is what happens after DTC testing. "The reason for testing is to individualize patient care, but is that happening?" Dr. Domchek asked. "There are limited data so far about DTC approaches, and most reported data is related to [single nucleotide polymorphism] testing, for which there is largely no evidence of significant health-related behavior change."

Further challenges are posed by patient interpretation of results, particularly in regard to an inappropriate understanding of variants of uncertain significance, moderate penetrance, limited genotyping, lack of data based on minority populations, and indeterminate negative results.

"We have to be really careful that people are getting guideline-driven care," she said. "It's important that patients and providers understand these tests and what they entail, as well as the implications of the results."

Disclosures: Dr. Domchek has disclosed that she has received honoraria from AstraZeneca Pharmaceuticals LP. Mr. Pilarski, Dr. Weiss, and Dr. Pal have disclosed that they have no financial interests, arrangements, affiliations, or commercial interests with the manufacturers of any products discussed in this article or their competitors.

Correspondence: Robert Pilarski, MS, LGC, MSW, Ohio State University, Division of Human Genetics, 2012 Kenny Road, Columbus, OH 43221. Email: robert.pilarski@osumc.edu;

Jennifer M. Weiss, MD, MS, University of Wisconsin School of Medicine and Public Health, 1685 Highland Avenue, Room 4200, Madison, WI 53705. Email: jmw@medicine.wisc.edu;

Susan M. Domchek, MD, University of Pennsylvania, 3400 Civic Center Boulevard, 10-150 South PCAM, Philadelphia, PA 19104.

Email: susan.domchek@pennmedicine.upenn.edu; and

Tuya Pal, MD, Vanderbilt University Medical Center, 1500 21st Ave South Suite 2500, Nashville, TN 37212. Email: tuya.pal@vumc.org

\section{References}

1. Lubbe SJ, Di Bernardo MC, Chandler IP, et al. Clinical implication of the colorectal cancer risk associated with MUTYH mutation. J Clin Oncol 2009; 27:3975-3980

2. Win AK, Dowty JG Cleary SP, et al. Risk of colorectal cancer for carriers of mutations in MUTYH, with and without a family history of cancer. Gastroenterology 2014;146:1208-1211.

3. Provenzale D, Gupta S, Ahnen DJ, et al. NCCN Clinical Practice Guidelines in Oncology: Genetic/Familial High-Risk Assessment: Colorectal. Version 3.2019. Accessed December 13, 2019. To view the most recent version, visit NCCN.org

4. Katona BW, Yurgelun MB, Garber JE, et al. A counseling framework for moderate-penetrance colorectal cancer susceptibility genes. Genet Med 2018;20:1324-1327.

5. Katona BW, Stadler ZK, Robson ME, et al. RE: BRCA1 and BRCA2 gene mutations and colorectal cancer risk: systematic review and meta-analysis. J Natl Cancer Inst 2019;111:522-523.

6. Kilbride MK, Joffe S. The new age of patient autonomy: implications for the patient-physician relationship. JAMA 2018;320: 1973-1974. 